\title{
Efficient Three-Dimensional Poisson Solvers in Open Rectangular Conducting Pipe
}

\author{
Ji Qiang* \\ Lawrence Berkeley National Laboratory \\ 1 Cycltron Road, Berkeley, CA 94720, USA
}

\begin{abstract}
Three-dimensional (3D) Poisson solver plays an important role in study of spacecharge effects on charged particle beam dynamics in particle accelerators. In this paper, we propose three new 3D Poisson solvers for a charged particle beam in an open rectangular conducting pipe. These three solvers include a spectral integrated Green function (IGF) solver, a 3D spectral solver, and a 3D integrated Green function solver. These solvers effectively handle the longitudinal open boundary condition using a finite computational domain that contains the beam itself. This saves the computational cost of using an extra larger longitudinal domain in order to set up an appropriate finite boundary condition. Using an integrated Green function also avoids the need to resolve rapid variation of the Green function inside the beam. The numerical operational cost of the spectral IGF solver and the 3D IGF solver scales as $O(N \log (N))$, where $N$ is the number of grid points. The cost of the $3 \mathrm{D}$ spectral solver scales as $O\left(N_{n} N\right)$, where $N_{n}$ is the maximum longitudinal mode number. We compare these three solvers using several numerical examples and discuss the advantageous regime of each solver in the physical application.
\end{abstract}

Keywords: Poisson equation, spectral method, Green function

\footnotetext{
${ }^{*}$ Corresponding author

Email address: jqiang@lbl.gov (Ji Qiang*)
}

Preprint submitted to Journal of ${ }^{A} T_{E} X$ Templates

January 19, 2016

(C) 2016. This manuscript version is made available under the Elsevier user license http://www.elsevier.com/open-access/userlicense/1.0/ 


\section{Introduction}

Three-dimensional Poisson's equation has important applications in physics, chemistry, biology and environment. In particle accelerator physics, the nonlinear space-charge effect due to Coulomb interactions of charged particles has significant impact to the particle beam quality in high intensity accelerators. It drives emittance growth of the beam and causes beam losses inside the accelerator. A natural way to include the space-charge effect in the simulation is through self-consistent particle-in-cell (PIC) method [1, 2, 3, 4, 5, 6]. In the PIC method, macroparticles are advanced step by step in phase space subject to both the external forces and the space-charge forces. Normally, at each step, the external forces can be quickly computed using the given external fields. The space-charge forces are calculated self-consistently using the charge density distribution at each step by solving the Poisson equation on a computational grid. Here, the charge density distribution is obtained from the deposition of discrete marcoparticles onto the computational grid using an assumed shape function. Solving the Poisson equation involves a large number of numerical operations and is much more computationally expensive than the external force calculation. An efficient Poisson solver will be of importance in the PIC simulation in order to quickly calculate the space-charge forces and to reduce the total simulation time.

In previous studies, a number of methods have been proposed to solve the Poisson equation inside a closed computational domain [7, 8, 9, 10, 11]. For fully three-dimensional open computational domain, an efficient Green's method using fast Fourier transforms (FFTs) and zero padding was used to solve the Poisson equation for the electric potential inside the beam [12, 13, 14]. This method was further improved to handle situation with large aspect ratio and with high order accuracy [15, 16, 17]. In some particle accelerators, the longitudinal size of the charged particle beam is larger than the aperture size of the pipe or the aperture size of the beam pipe is small, the effects of conducting pipe on the beam are not negligible. Figure 1 shows a schematic plot of a charged particle 
beam inside an open rectangular conducting pipe. The electric potential in the Poisson equation for the beam has a finite boundary condition in the transverse plane and an open boundary condition in the longitudinal direction. A brute force method is to make the longitudinal computational domain large enough so that the potential vanishes at the edge computational domain. The Poisson equation can be solved within a closed computational domain subject to the 3D Dirichlet boundary condition. However, using a large extra computational domain beyond the beam may waste computing resource since only the electric potential/field inside the beam is needed to advance charged particles in the PIC simulation. An efficient method to handle the longitudinal open boundary condition was proposed in our previous study using a boundary matching procedure [18]. However, this method uses a finite difference scheme for the longitudinal discretization. For a long bunch with very large longitudinal to transverse aspect ratio, (e.g. $>100$ ), the use of a finite difference method in the longitudinal direction may not be computationally efficient. In reference [19], a Hermite-Gaussian expansion was proposed to handle longitudinal open boundary condition in a round conducting pipe. For a rectangular conducting pipe, a Green's function method was proposed by Ryne to effectively handle the longitudinal open boundary condition [20]. This method uses a standard Green function in the transverse plane and an integrated Green function in the longitudinal direction. The solution is then calculated using an FFT based method with zero padding. In this paper, we propose three new alternative methods to solve the Poisson equation in an open rectangular conducting pipe. These new methods are spectral integrated Green function method, 3D spectral method, and 3D integrated Green function method. To the best of our knowledge, none of these methods was reported before to solve the 3D Poisson equation in an open rectangular conducting pipe. All three methods use a computational domain that longitudinally contains only the beam itself. No extra computational domain is needed in the longitudinal direction in order to meet the open boundary conditions on both sides of the beam. These new solvers effectively handle the longitudinal open boundary conditions within a finite computational domain 


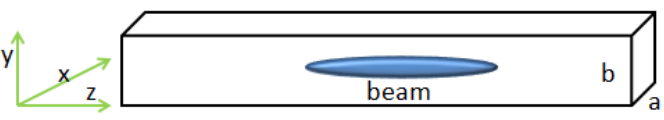

Figure 1: A schematic plot of a charged particle beam inside an open rectangular conducting pipe.

using a spectral method and an integrated Green function method. The integrated Green function method also provides an efficient way to solve the Poisson equation inside a computational domain with a large longitudinal-to-transverse aspect ratio in comparison to the conventional Green's function method.

The organization of this paper is as follows: We will present the three numerical methods in Section 2. We give several numerical examples in Section 3. Conclusions are drawn in Section 4.

\section{Numerical Methods}

For a charged particle beam inside an open rectangular conducting pipe, we write the three-dimensional Poisson equation as:

$$
\frac{\partial^{2} \phi}{\partial x^{2}}+\frac{\partial^{2} \phi}{\partial y^{2}}+\frac{\partial^{2} \phi}{\partial z^{2}}=-\frac{\rho}{\epsilon_{0}}
$$

where, $\phi$ denotes the electric potential inside the beam, $\rho$ the charge density distribution of the beam, $x, y$ and $z$ denote the horizontal, vertical, and longitudinal coordinates respectively. The boundary conditions for the electric potential in the open rectangular perfect conducting pipe are:

$$
\begin{aligned}
\phi(x=0, y, z) & =0 \\
\phi(x=a, y, z) & =0 \\
\phi(x, y=0, z) & =0 \\
\phi(x, y=b, z) & =0 \\
\phi(x, y, z= \pm \infty) & =0
\end{aligned}
$$

where $a$ is the horizontal width of the pipe and $b$ is the vertical width of the pipe. In the following, we propose three efficient numerical methods to solve 
the Poisson Eq. 1 subject to above boundary conditions.

\subsection{Spectral Integrated Green Function Method}

Given the boundary conditions in Eq. 2-6 the electric potential $\phi$ and the source term $\rho$ can be approximated using two sine functions as [21, 22, 23, 24]:

$$
\begin{aligned}
& \rho(x, y, z)=\sum_{l=1}^{N_{l}} \sum_{m=1}^{N_{m}} \rho^{l m}(z) \sin \left(\alpha_{l} x\right) \sin \left(\beta_{m} y\right) \\
& \phi(x, y, z)=\sum_{l=1}^{N_{l}} \sum_{m=1}^{N_{m}} \phi^{l m}(z) \sin \left(\alpha_{l} x\right) \sin \left(\beta_{m} y\right)
\end{aligned}
$$

where

$$
\begin{aligned}
& \rho^{l m}(x, y, z)=\frac{4}{a b} \int_{0}^{a} \int_{0}^{b} \rho(x, y, z) \sin \left(\alpha_{l} x\right) \sin \left(\beta_{m} y\right) d x d y \\
& \phi^{l m}(x, y, z)=\frac{4}{a b} \int_{0}^{a} \int_{0}^{b} \phi(x, y, z) \sin \left(\alpha_{l} x\right) \sin \left(\beta_{m} y\right) d x d y
\end{aligned}
$$

where $\alpha_{l}=l \pi / a$ and $\beta_{m}=m \pi / b$. The above approximation follows a numerical spectral Galerkin method since each basis function satisfies the transverse boundary conditions on the wall. For a smooth analytical function, this spectral approximation has an accuracy with the numerical error that scales as $O(\exp (-c N))$ with $c>0$ and $N$ is the order of the basis function used in the approximation. Substituting above expansions into the Poisson equation and making use of the orthonormal conditions of the sine functions, we obtain

$$
\frac{\partial^{2} \phi^{l m}(z)}{\partial z^{2}}-\gamma_{l m}^{2} \phi^{l m}(z)=-\frac{\rho^{l m}}{\epsilon_{0}}
$$

where $\gamma_{l m}^{2}=\alpha_{l}^{2}+\beta_{m}^{2}$. The above ordinary differential equation for each mode $l m$ can be solved using a Green function method. This solution can be written as:

$$
\phi^{l m}(z)=\frac{1}{2 \gamma_{l m} \epsilon_{0}} \int G_{l m}\left(z-z^{\prime}\right) \rho^{l m}\left(z^{\prime}\right) d z^{\prime}
$$

where the Green function $G_{l m}$ is given by:

$$
G_{l m}\left(z-z^{\prime}\right)=\exp \left(-\gamma_{l m}\left|z-z^{\prime}\right|\right)
$$


The above convolution integral can be discretized on a mesh that longitudinally contains only the beam. The discrete electric potential on an equidistant grid point $z_{i}, i=1,2, \cdots, N_{z}$ is given as:

$$
\phi^{l m}\left(z_{i}\right)=\frac{h_{z}}{2 \gamma_{l m} \epsilon_{0}} \sum_{j=1}^{N_{z}} G_{l m}\left(z_{i}-z_{j}\right) \rho^{l m}\left(z_{j}\right)
$$

where $h_{z}=\left(z_{\max }-z_{\min }\right) /\left(N_{z}-1\right), z_{i}=z_{\min }+(i-1) h_{z}, z_{\min }$ and $z_{\max }$ are the minimum and the maximum locations of the beam along the $z$ direction respectively, and $N_{z}$ is the number of longitudinal grid points. The above summation corresponds to a numerical trapezoidal rule approximation to the integral and has an accuracy of $O\left(1 / N_{z}^{2}\right)$. The direct brute-force calculation of above discrete convolution summation for all $N_{z}$ grid points takes $O\left(N_{z}^{2}\right)$ operations. Fortunately, by using the FFT with the zero padding method, the computational cost of the summation for all $N_{z}$ grid points can be reduced into $O\left(N_{z} \log \left(N_{z}\right)\right)$ for each transverse mode $l m$.

The Green function given in Eq. 13 exponentially decreases with the increase of the separation between two grid points. In the numerical calculation of the integral Eq. 12, resolving the Green function may not be necessary if the variation of beam density along $z$ is slower than the decreasing rate of the Green function. The convolution integral Eq. 12 is broken into a summation of a number of small interval convolutions and can be rewritten as:

$$
\phi^{l m}(z)=\frac{1}{2 \gamma_{l m} \epsilon_{0}} \sum_{j} \int_{z_{j}-h_{z} / 2}^{z_{j}+h_{z} / 2} G_{l m}\left(z-z^{\prime}\right) \rho^{l m}\left(z^{\prime}\right) d z^{\prime}
$$

where $j=1,2, \cdots, N_{z}$. If we assume that the charge density $\rho^{l m}$ stays constant within the interval $\left[z_{j}-h_{z} / 2, z_{j}+h_{z} / 2\right]$, the above equation can be reduced into:

$$
\phi^{l m}(z)=\frac{1}{2 \gamma_{l m} \epsilon_{0}} \sum_{j} G_{l m}^{i n t}\left(z-z_{j}\right) \rho^{l m}\left(z_{j}\right)
$$

where

$$
G_{l m}^{i n t}\left(z-z_{j}\right)=\int_{z_{j}-h_{z} / 2}^{z_{j}+h_{z} / 2} G_{l m}(z-t) d t
$$


Substituting Eq.13]into above equation, we obtain the integrated Green function as:

$G_{l m}^{i n t}\left(z_{i}-z_{j}\right)=\left\{\begin{array}{l}\frac{\exp \left(-\gamma_{l m}\left|z_{i}-z_{j}\right|\right)}{\gamma_{l m}}\left(\exp \left(\gamma_{l m} h_{z} / 2\right)-\exp \left(-\gamma_{l m} h_{z} / 2\right)\right), \text { if } i \neq j \\ \frac{2}{\gamma_{l m}}\left(1-\exp \left(-\gamma_{l m} h_{z} / 2\right)\right) \text { otherwise }\end{array}\right.$

Using the integrated Green function $G_{l m}^{i n t}$, the convolution summation Eq.[16]can be calculated using the same FFT with the zero padding method as the standard Green function method. The numerical error in the above approximation scales as $O\left(h_{z}^{2}\right)$. The advantage of this method is that the fast decrease of the Green function does not need to be resolved in the numerical approximation to the convolution integral Eq. 12, which can significantly save computational resource in some applications.

The numerical calculation of the sine function transform in both $x$ and $y$ directions can be done efficiently using the FFT method. The computational cost in transverse $x$ and $y$ dimensions scales as $O\left(N_{x} N_{y} N_{z}\left(\log \left(N_{x} N_{y}\right)\right)\right.$. Here, we assume that the transverse mode numbers $N_{l}=N_{x}$ and $N_{m}=N_{y}$. Using the zero padding and the FFT for the discrete convolution summation of each transverse mode, the cost to compute the convolution along $z$ direction scales as $O\left(N_{x} N_{y} N_{z} \log \left(N_{z}\right)\right)$. This results in a total computational cost to solve the 3D Poisson equation in an open conducting pipe scaling as $O\left(N_{x} N_{y} N_{z} \log \left(N_{x} N_{y} N_{z}\right)\right)$.

\subsection{D Spectral Method}

In many accelerator physics application, the longitudinal density distribution of the charged particle beam has a Gaussian distribution. This suggests that the ordinary differential Eq. 11 can also be solved efficiently using a HermiteGaussian series expansion, which naturally satisfies the open boundary condition (Eq. 6) in the longitudinal $z$ direction.

The charge density $\rho$ and the electric potential $\phi$ for each transverse mode 
$l m$ can be approximated as [21, 22, 24]:

$$
\begin{aligned}
\rho^{l m}(z) & =\sum_{n=0}^{N_{n}} \rho_{n}^{l m} \mathcal{H}_{n}(z) \\
\phi^{l m}(z) & =\sum_{n=0}^{N_{n}} \phi_{n}^{l m} \mathcal{H}_{n}(z)
\end{aligned}
$$

where the scaled Hermite-Gaussian function $\mathcal{H}_{n}$ is defined as:

$$
\mathcal{H}_{n}(z)=H_{n}\left(\frac{z}{A}\right) \exp \left(-\frac{1}{2} \frac{z^{2}}{A^{2}}\right)
$$

where $A$ is a longitudinal scaling constant, which can be set as the beam longitudinal root mean square (RMS) size $\sigma_{z}, H_{n}$ is the $n^{\text {th }}$ order Hermite polynomial with properties: $H_{0}(z)=1, H_{1}(z)=2 z, \cdots, H_{n}(z)=2 z H_{n-1}-2(n-1) H_{n-2}$. The scaled Hermite-Gaussian function $\mathcal{H}$ has the properties:

$$
\int_{-\infty}^{\infty} \mathcal{H}_{n}(z) \mathcal{H}_{m}(z) d z=2^{n} n ! \sqrt{\pi} A \delta_{n m}
$$

and

$$
\frac{\partial^{2} \mathcal{H}_{n}}{\partial z^{2}}=\frac{1}{4 A^{2}} \mathcal{H}_{n+2}+\frac{n(n-1)}{A^{2}} \mathcal{H}_{n-2}-\frac{2 n+1}{2 A^{2}} \mathcal{H}_{n}
$$

where $\delta_{m n}=1$ for $m=n$ and $\delta_{m n}=0$ for $m \neq n$. The expansion coefficients $\rho_{n}$ and $\phi_{n}$ can be obtained from

$$
\begin{aligned}
\rho_{n}^{l m} & =\frac{1}{2^{n} n ! \sqrt{\pi} A} \int_{-\infty}^{\infty} \rho^{l m}(z) \mathcal{H}_{n}(z) d z \\
\phi_{n}^{l m} & =\frac{1}{2^{n} n ! \sqrt{\pi} A} \int_{-\infty}^{\infty} \phi^{l m}(z) \mathcal{H}_{n}(z) d z
\end{aligned}
$$

The above approximation also follows the spectral Galerkin method since the Hermite-Gaussian basis function satisfies the longitudinal open boundary condition naturally. For a smooth function, this results in the error in above approximation going to zero exponentially with respect to the number of the basis function. Substituting the functions $\rho$ and $\phi$ into the Eq. 11, and using the orthogonality of the scaled Hermite-Gaussian functions Eq. 22, the Poisson equation is reduced into a group of linear algebraic equations:

$$
\frac{1}{4} \phi_{n-2}^{l m}-\left(\frac{1}{2}(2 n+1)+\gamma_{l m}^{2} A^{2}\right) \phi_{n}^{l m}+(n+2)(n+1) \phi_{n+2}^{l m}=-A^{2} \rho_{n}^{l m} / \epsilon_{0}
$$


where $n=1,2, \cdots, N_{n}$ and $N_{n}$ is the number of Hermite-Gaussian modes, and $\phi_{-1}^{l m}=\phi_{0}^{l m}=\phi_{N_{n}+1}^{l m}=\phi_{N_{n}+2}^{l m}=0$. This group of algebraic equations is a band-limited matrix equation, which can be solved effectively using direct Gaussian elimination with a numerical operation cost of $O\left(N_{n}\right)$ for each transverse mode $\mathrm{lm}$. This results in a total cost to solve above equations scaling as $O\left(N_{l} N_{m} N_{n}\right)$ for all modes. The computational cost of the sine transform scales as $O\left(N_{x} N_{y} N_{z} \log \left(N_{x} N_{y}\right)\right)$. The computing of the Hermite-Gaussian expansion coefficients is more expensive and scales as $O\left(N_{l} N_{m} N_{n} N_{z}\right)$. If the number of the Hermite-Gaussian modes can be controlled within a reasonable limit by taking advantage of the high order accuracy of the spectral method, this method can still be very efficient. Another advantage of this method is that it provides a natural smoothing of electric potential in the self-consistent PIC simulation by removing the high frequency modes in the expansion.

\subsection{D Integrated Green Function Method}

Another method to solve the 3D Poisson equation inside the open rectangular pipe is to use an integrated Green function method directly. In reference [20], a transverse standard Green function and a longitudinal integrated Green function method was used to solve the Poisson equation in the open conducting pipe. In this paper, we extend that method into a fully 3D integrated Green function method. Using a 3D integrated Green function saves the computational cost in order to resolve the Green function variation on numerical grids. This method has also the advantage of using a computational domain that contains only the beam itself instead of the whole transverse pipe cross-section.

In Section 2.1, we expand the solution of electric potential and the charge density transversely using two sine functions and obtain the longitudinal $z$ dependent solution using a Green's function method. By substituting the Eqs. 9 and 13 into the Eq. 12, then the Eq. 12 into the Eq. 8, we obtain the solution 
of the 3D Poisson equation inside an open rectangular pipe as:

$$
\begin{array}{r}
\phi(x, y, z)=\frac{2}{a b \epsilon_{0}} \sum_{l=1}^{\infty} \sum_{m=1}^{\infty} \frac{1}{\gamma_{l m}} \sin \left(\alpha_{l} x\right) \sin \left(\beta_{m} y\right) \int_{x_{\min }}^{x_{\max }} \int_{y_{\min }}^{y_{\max }} \int_{z_{\min }}^{z_{\max }} \sin \left(\alpha_{l} x^{\prime}\right) \sin \left(\beta_{m} y^{\prime}\right) \times \\
\exp \left(-\gamma_{l m}\left|z-z^{\prime}\right|\right) \rho\left(x^{\prime}, y^{\prime}, z^{\prime}\right) d x^{\prime} d y^{\prime} d z^{\prime}
\end{array}
$$

A direct brute-force calculation of the electric potential following above equation on all $N_{x} N_{y} N_{z}$ grid points takes $O\left(\left(N_{x} N_{y} N_{z}\right)^{2}\right)$ operations. Meanwhile, this equation can be rewritten as:

$$
\begin{aligned}
\phi(x, y, z)= & \frac{1}{2 a b \epsilon_{0}} \int_{x_{\min }}^{x_{\max }} \int_{y_{\min }}^{y_{\max }} \int_{z_{\min }}^{z_{\max }} \sum_{l=1}^{\infty} \sum_{m=1}^{\infty} \frac{1}{\gamma_{l m}}\left[\cos \left(\alpha_{l}\left(x-x^{\prime}\right)\right)-\cos \left(\alpha_{l}\left(x+x^{\prime}\right)\right)\right] \times \\
& \left(\cos \left(\beta_{m}\left(y-y^{\prime}\right)\right)-\cos \left(\beta_{m}\left(y+y^{\prime}\right)\right)\right) \exp \left(-\gamma_{l m}\left|z-z^{\prime}\right|\right) \rho\left(x^{\prime}, y^{\prime}, z^{\prime}\right) d x^{\prime} d y^{\prime} d z^{\prime}
\end{aligned}
$$

Following the same idea of the preceding subsection, we can define a threedimensional integrated Green function as:

$$
\begin{array}{r}
G_{3 D}^{i n t}\left(x, x^{\prime}, y, y^{\prime}, z, z^{\prime}\right)=\frac{1}{2 a b \epsilon_{0}}\left(R\left(x-x^{\prime}, y-y^{\prime}, z-z^{\prime}\right)-\right. \\
\left.R\left(x-x^{\prime}, y+y^{\prime}, z-z^{\prime}\right)-R\left(x+x^{\prime}, y-y^{\prime}, z-z^{\prime}\right)+R\left(x+x^{\prime}, y+y^{\prime}, z-z^{\prime}\right)\right)
\end{array}
$$

where

$$
\begin{array}{r}
R(u, v, w)=\sum_{l=1}^{\infty} \sum_{m=1}^{\infty} \frac{1}{\alpha_{l} \beta_{m} \gamma_{l m}}\left[\sin \left(\alpha_{l}\left(u-h_{x} / 2\right)\right)-\sin \left(\alpha_{l}\left(u+h_{x} / 2\right)\right)\right] \times \\
\left(\sin \left(\beta_{m}\left(v-h_{y} / 2\right)\right)-\sin \left(\beta_{m}\left(v+h_{y} / 2\right)\right)\right) G_{l m}^{i n t}(w)
\end{array}
$$

where $h_{x}=\left(x_{\max }-x_{\min }\right) /\left(N_{x}-1\right)$ and $h_{y}=\left(y_{\max }-y_{\min }\right) /\left(N_{y}-1\right)$. Using the extended trapezoidal quadrature rule in $3 \mathrm{D}$, the electric potential on a grid $(i, j, k)$ can be approximated as:

$$
\phi\left(x_{i}, y_{j}, z_{k}\right)=\sum_{i=1}^{N_{x}} \sum_{j=1}^{N_{y}} \sum_{k=1}^{N_{z}} G_{3 D}^{i n t}\left(x_{i}, y_{j}, z_{k}, x_{i}^{\prime}, y_{j}^{\prime}, z_{k}^{\prime}\right) \rho\left(x_{i}^{\prime}, y_{j}^{\prime}, z_{k}^{\prime}\right)
$$

The numerical error in above approximation scales as $O\left(1 / N_{x}^{2}+1 / N_{y}^{2}+1 / N_{z}^{2}\right)$. The above summation can also be calculated efficiently on a doubled computational domain using an FFT method with zero padding method. In order to compute this summation using the FFT-based method, besides the direct convolution term $R\left(x-x^{\prime}, y-y^{\prime}, z-z^{\prime}\right) \rho\left(x^{\prime}, y^{\prime}, z^{\prime}\right)$, there are also terms that 
contain auto-correlations in $R\left(x-x^{\prime}, y+y^{\prime}, z-z^{\prime}\right), R\left(x+x^{\prime}, y-y^{\prime}, z-z^{\prime}\right)$, and $R\left(x+x^{\prime}, y+y^{\prime}, z-z^{\prime}\right)$. It turns out that those auto-correlations can be handled in a similar way to the convolution term except that the backward/forward FFT is used in the dimension with auto-correlation while the forward/backward FFT is used in the dimension with convolution [20]. The total operational cost for the summation in Eq. 31 scales as $O\left(N_{x} N_{y} N_{z} \log \left(N_{x} N_{y} N_{z}\right)\right)$ for all $N_{x} N_{y} N_{z}$ grid points.

\section{Numerical Examples}

In the following, we present several numerical examples for the above proposed algorithms. Here, we assume that the charged particle beam has a 3D Gaussian density distribution as:

$$
\rho(x, y, z)=\exp \left(-\frac{\left(x-x_{0}\right)^{2}}{2 \sigma_{x}^{2}}-\frac{\left(y-y_{0}\right)^{2}}{2 \sigma_{y}^{2}}-\frac{\left(z-z_{0}\right)^{2}}{2 \sigma_{z}^{2}}\right)
$$

where $\sigma_{x}, \sigma_{y}$, and $\sigma_{z}$ denote RMS sizes of the beam, and $x_{0}, y_{0}$, and $z_{0}$ denote the centroid of the beam.

In the first example, we study the effects of longitudinal-to-transverse aspect ratio on the accuracy of the numerical solution. We assume that the pipe transverse aperture sizes $a=b=2$, transverse RMS beam sizes $\sigma_{x}=\sigma_{y}=1 / 6$, and the longitudinal RMS size $\sigma_{z}=1 / 6$ that results in an aspect ratio of $A=1$, and the $\sigma_{z}=16.67$ for an aspect ratio of $A=100$. The computational grid used in this example is $65 \times 65 \times 64$, and the maximum transverse and longitudinal modes $N_{l}=N_{m}=N_{n}=64$. Figure 2 shows the electric potential solution onaxis(red lines) and off-axis(green lines) as a function of the longitudinal distance using the spectral standard Green function method and the spectral integrated Green function method. It is seen that for small aspect ratio beam $(A=1)$, the two methods agree with each other very well. However, for large aspect ratio beam $(A=100)$, the integrated Green function solutions are very different from those obtained from the standard Green function solutions. The standard Green function method significantly over-predicts the on-axis and the off-axis 


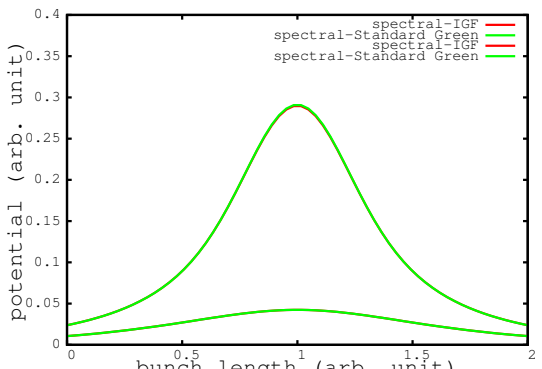

bunch length (arb. unit)

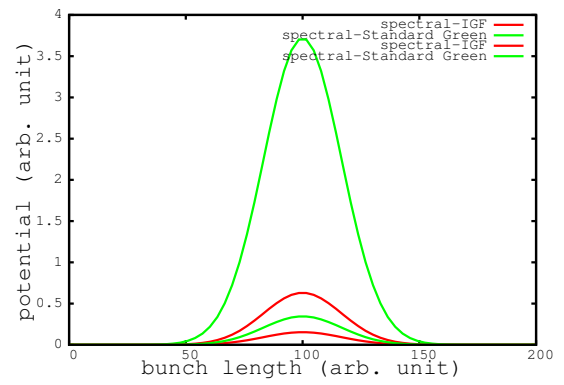

Figure 2: The on-axis potential solution $\phi(a / 2, b / 2, z)$ and off-axis $\phi(a / 4, b / 4, z)$ potential solution as function of $z$ from the spectral standard Green function method (green) and from the spectral IGF method (red) for a beam with an aspect ratio 1 (left) and an aspect ratio 100 (right).

electric potentials. This is because the standard Green function method in the longitudinal direction fails to resolve the fast variation of the Green function in Eq. 13 as the bunch length increases in the large aspect ratio case and the longitudinal mesh size $\left(h_{z}\right)$ increases with fixed longitudinal grid points.

In the second numerical example, we would like to compare the solutions from the above three numerical methods with the analytical solutions using the same numerical parameters as the preceding example with the aspect ratio $A=100$. The analytical solutions were obtained directly from the Green's function solution Eq. 27 based on a high precision calculation of the integrals and summations using $2001 \times 2001 \times 20001$ grid points and $32 \times 32$ transverse modes. Figure 3 shows the electric potential solutions and the relative errors along the center horizontal axis from the above three numerical methods (the spectral integrated Green function (green line), the 3D spectral method (blue line), the 3D integrated Green function method (pink line)) and the analytical solution (red line). All three numerical methods produce solutions with relative errors below $0.1 \%$. The $3 \mathrm{D}$ spectral method solution has the least relative errors as expected. The 3D integrated Green function method has the largest relative errors but is still below $0.1 \%$. Figure 4 shows the electric potential solutions and the relative errors along center longitudinal axis in this example using the three numerical methods together with the analytical solution. Again, all three 

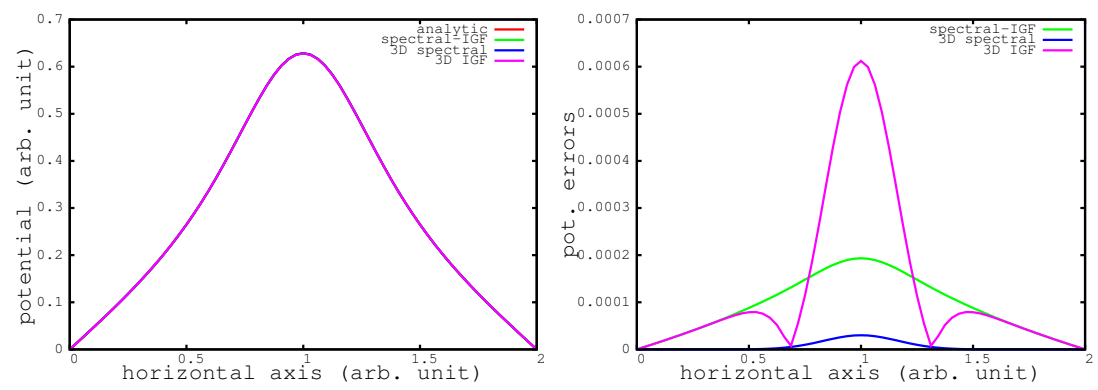

Figure 3: The electric potential solutions (left) and relative errors (right) along the horizontal axis from the three proposed numerical algorithms using a $65 \times 65 \times 64$ computational grid and from the analytical solution.
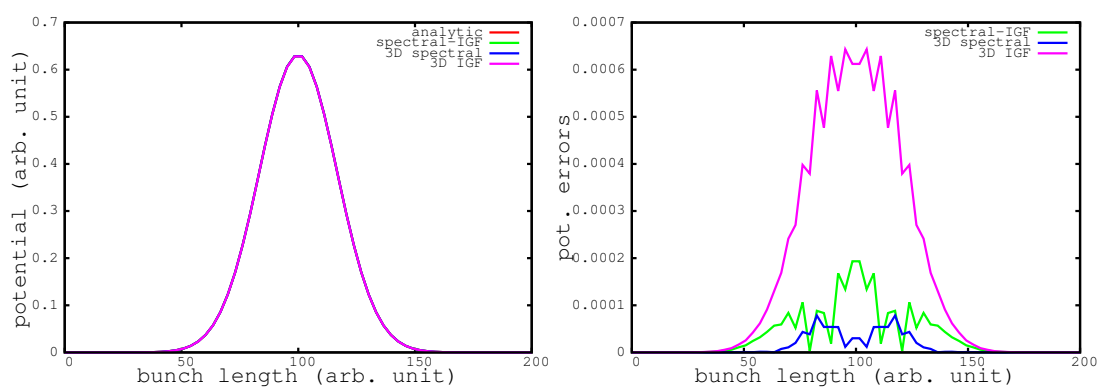

Figure 4: The electric potential solutions (left) and relative errors (right) along the longitudinal axis from the three proposed numerical algorithms using a $65 \times 65 \times 64$ computational grid and from the analytical solution. 
Figure 5: A schematic plot of a long small transverse size beam inside a rectangular conducting pipe together with the computational domain for the 3D integrated Green function method.

methods give a good approximation to the analytical solution with the maximum relative error below $0.1 \%$. The $3 \mathrm{D}$ spectral method shows the least relative error among the three methods.

The 3D integrated Green function method has larger relative errors than the other two methods. It is also more computational expensive than the other two methods since the calculation of the integrated Green function itself involves double summations with respect to the number of transverse modes. However, it has the advantage that the convolution integral contains the beam itself in a 3D computational domain. For example, in the application with a small, long beam inside a rectangular conducting pipe as shown in Fig. [5 the computational domain for the 3D integrated Green function method needs only to contain the beam instead of the entire transverse cross section of the conducting pipe. This helps to improve the numerical resolution and accuracy of the solution. In the following numerical example, we assume a Gaussian distribution beam with $\sigma_{x}=\sigma_{y}=1 / 48$ and $\sigma_{z}=66.67$, and pipe aperture $a=b=2$. This beam has a very large aspect ratio of 3200. Such a large aspect ratio beam can be found in some storage ring accelerators. The computational grid used in this example is $65 \times 65 \times 64$, and the maximum transverse and longitudinal mode number $N_{l}=N_{m}=N_{n}=64$. Figure 6 shows the electric potential distribution solutions and relative errors on center axis along the longitudinal $z$ direction from the analytical solution and from the three numerical methods. It is seen that the 3D integrated Green function method has the least error among the three solutions. This is because that this method uses a computational domain contains the beam itself, while the other two methods use a computational grid that covers the entire transverse cross-section of the pipe, hence, less numerical 

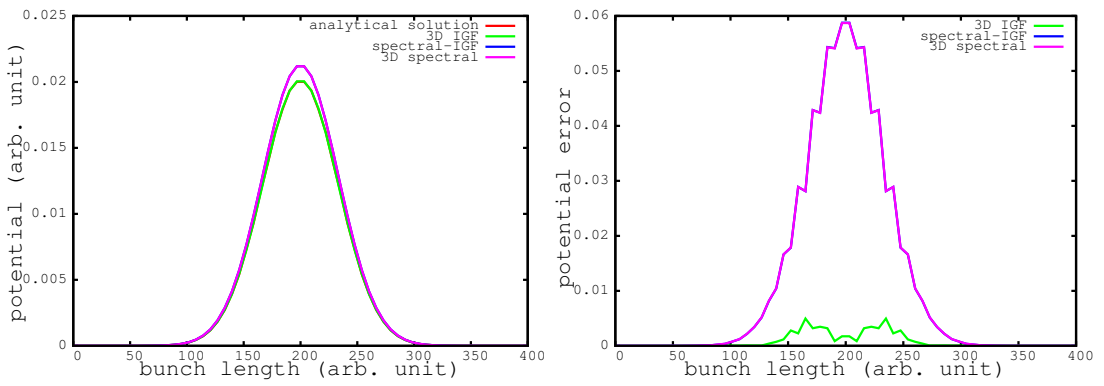

Figure 6: The electric potential solutions (left) and relative errors (right) along the longitudinal axis from the three proposed numerical algorithms and from the analytical solution for a long small transverse size beam.

resolution of the beam itself. The two solutions from the spectral-integrated Green function method and the 3D spectral method show similar larger errors than that from the 3D integrated Green function method. This is due to the fact that the poor transverse resolution in both methods results in these large errors.

\section{Conclusions}

In this paper, we proposed three new 3D Poisson solvers to calculate the electric potential in the charged particle beam inside an open conducting rectangular pipe. Those three Poisson solvers effectively save the computing resource by using a computational domain that longitudinally contains the beam itself. Using an integrated Green function in two solvers also avoids the need to resolve rapid variation of the Green function inside the beam and saves the computational cost of using a large number of grid points in the solution. The spectral integrated Green function solver and the 3D integrated Green function solver have a computational complexity of $O(N \log (N))$, where $N$ is the total number of grid points. The computational cost of the 3D spectral solver scales as $O\left(N_{n} N\right)$, where $N_{n}$ is the number of Hermite-Gaussian modes used in the solution. Given the fast convergence rate of the spectral solver, the mode number might be kept small. In scaling estimation, the spectral integrated Green function Poisson solver and the 3D integrated Green function Poisson solver 
are more efficient than the 3D spectral Poisson solver. In practical application, the 3D integrated Green function Poisson solver is most time consuming due to the double summations in the Green function. However, it has the advantage that the computational domain only needs to contain the beam itself in both transverse and longitudinal directions. This saves computational cost when the transverse size of the beam is much smaller than the transverse aperture of the pipe. The 3D spectral solver has an extra cost factor depending on the HermiteGaussian mode number. However, this solver normally has less numerical errors and can also provide smooth electric potential solution even if the charge density function contains numerical noise from the discrete macroparticle deposition in the PIC simulation. The spectral integrated Green function solver has a numerical accuracy between the 3D spectral solver and the 3D integrated Green function solver, but a favorable computational cost scaling.

\section{Acknowledgment}

The work was supported by the U.S. Department of Energy under Contract No. DE-AC02-05CH11231. This research used computer resources at the National Energy Research Scientific Computing Center and at the National Center for Computational Sciences.

\section{References}

[1] A. Friedman, D. P. Grote and I. Haber, Phys. Fluids B 4, 2203 (1992).

[2] T. Takeda, J. H. Billen, "Recent developments of the accelerator design code PARMILA," Proceedings of the XIX International Linac Conference, Chicago, IL, August 1998, pp. 156-158.

[3] J. Qiang, R. D. Ryne, S. Habib, V. Decyk, J. Comput. Phys. 163, 434 (2000).

[4] J. Qiang, S. Lidia, R. D. Ryne, and C. Limborg-Deprey, Phys. Rev. ST Accel. Beams 9, 044204 (2006). 
[5] J. Amundson, P. Spentzouris, J.Qiang and R. Ryne, J. Comp. Phys. vol. 211, 229 (2006).

[6] A. Adelmann, et al., The OPAL (Object Oriented Parallel Accelerator Library) Framework, Technical Report PSI-PR-08-02, Paul Scherrer Institut, 20082014, http://amas.web.psi.ch/docs/opal/opal_user_guide.pdf.

[7] D. B. Haidvogel and T. Zang, J. Comput. Phys. 30, 167 (1979).

[8] S. Ohring, J. Comput. Phys. 50, 307 (1983).

[9] H. Dang-Vu and C. Delcarte, J. Comput. Phys. 104, 211 (1993).

[10] E. Braverman, M. Israeli, A. Averbuch, and L. Vozovoi, J. Comput. Phys. 144, (1998).

[11] L. Plagne and J. Berthou, J. Comput. Phys. 157, 419 (2000).

[12] J.W. Eastwood, D.R.K. Brownrigg, J. Comput. Phys. 32 (1979) 24.

[13] R.W. Hockney, J.W. Eastwood, Computer Simulation Using Particles, Adam Hilger, New York, 1988.

[14] W.H. Press, B.P. Flannery, S.A. Teukolsky, W.T. Vetterling, Numerical Recipes in FORTRAN: The Art of Scientific Computing, 2nd ed., Cambridge University Press, Cambridge, England, 1992.

[15] J. Qiang, S. Lidia, R. D. Ryne, and C. Limborg-Deprey, Phys. Rev. ST Accel. Beams 10, 129901 (2007).

[16] L. Genovese, T. Deutsch, A. Neelov, S. Godecker, G. Beylkin, J. Chem. Phys. 125, 074105 (2006).

[17] M. M. Hejlesen, J. T. Rasmussen, P. Chatelain, J. H. Walther, J. Comput. Phys. 252, 458 (2013).

[18] J. Qiang and R. D. Ryne, Comp. Phys. Comm. 138, 18 (2001).

[19] J. Qiang and R. Gluckstern, Comp. Phys. Comm. 160, 120, (2004). 
[20] R. D. Ryne, "On FFT based convolutions and correlations, with application to solving Poisson's equation in an open rectangular pipe," arXiv:1111.4971v1, 2011.

[21] D. Gottlieb and S. A. Orszag, Numerical Analysis of Spectral Methods: Theory and Applications, Society for Industrial and Applied Mathematics, 1977.

[22] D. Funaro, Polynomial Approximation of Differential Equations, SpringerVerlag, 1992.

[23] B. Fornberg, A Practical Guide to Pseudospectral Methods, Cambridge University Press, 1998.

[24] J. Boyd, Chebyshev and Fourier Spectral Methods, Dover Publications, Inc. 2000 . 